\title{
Zur Koordinations-Isomerie von Kobalt-methyl-Faktor I b und Kobalt-methyl-cobalamin ${ }^{1}$
}

\author{
Wilhelm Friedrich und MatthäUs Moskophidis ${ }^{2}$ \\ Physiologisch-Chemisches Institut der Universität Hamburg \\ (Z. Naturforsch. 25 b, 979-983 [1970]; eingegangen am 8. April 1970)
}

\begin{abstract}
Methylierung von Faktor I b am Kobalt ergibt zwei Diastereoisomere im Mengenverhältnis von $1: 1$, die sich säulenchromatographisch mittels Sephadex-DEAE A-50-CN ${ }^{\ominus}$ bei $0{ }^{\circ} \mathrm{C}$ sehr gut trennen lassen. Das schneller wandernde Isomer b (Methylgruppe „oben“) hat das gleiche Absorptionsspektrum wie Kobalt-methyl-cobinamid. Das langsamer wandernde Isomer a (Methylgruppe „unten") unterscheidet sich im Absorptionsspekrum nicht von der Kobalt-methyl-cobyrsäure a. Die Isomeren a und b lassen sich durch Licht bzw. Wärme $\left(80-100^{\circ} \mathrm{C}\right)$ ineinander umwandeln, wobei ein spezifisches Gleichgewicht erreicht werden kann. Belichtung führt zu $30 \%$ a (neben $70 \%$ b), Erhitzung zu $11 \%$ a (neben $89 \%$ b). Die Isomerisierung verläuft mit maximaler Geschwindigkeit in Wasser, nach Sättigung mit CO. Praktisch keine Isomerisierung wird bei Belichtung in $\mathrm{O}_{2}$-Atmosphäre sowie in alkoholischen Medien beobachtet. In der Wärme in $\mathrm{O}_{2}$-Atmosphäre ist die Isomerisierung sehr langsam.
\end{abstract}

Wir fanden vor kurzem, daß einige partialsynthetisch dargestellte Kobalt-methyl-corrinoide sich chromatographisch zu zwei isomeren Formen auftrennen lassen, die sich nach den bisherigen Befunden durch die Lage der Methylgruppe („oben“ bzw. „unten“) unterscheiden ${ }^{3-6}$. Alle diese Corrinoide besitzen an der „unteren“ Molekülseite eine saure Gruppe: ein Carboxyl (in der Cobyrsäure und in den Cobinamidmonocarbonsäuren) bzw. eine monobasige Phosphorsäuregruppe (im Cobalamin). Keine Isomerentrennung wurde bisher beim Kobalt-methyl-cobinamid, welchem eine derartige saure Gruppe fehlt, erzielt, obwohl hier eine solche Isomerie vorliegen dürfte.

Faktor Ib (Cobinamid-phosphoribose), ein aus

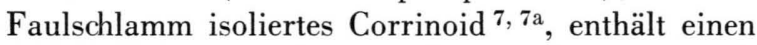
monobasigen Phosphorsäure-Rest und dürfte somit in die Gruppe der Corrinoide gehören, deren Kobalt-methyl-Derivate auf eine einfache Weise in zwei isomere Formen auftrennbar sind. Diese Annahme bestätigte sich. Es zeigte sich darüber hinaus völlig unerwartet, daß hier das Mengenverhältnis der beiden Isomeren $1: 1$ ist, also anders als bei den

1 Herrn Prof. Dr. K. Bernhauer zum 70. Geburtstag gewidmet.

2 Unter Mitarbeit von Frau S. A. Bagal.

3 W. Friedrich u. J. P. Nordmeyer, Z. Naturforsch. 23 b, 1119 [1968].

4 W. Friedrich u. R. Messerschmidt, Z. Naturforsch. 24 b, 465 [1969].

5 W. Friedrich u. J. P. Nordmeyer, Z. Naturforschg. 24 b, 588 [1969].

6 Vorausgehende Mitt., W. Friedrich u. R. Messerschmidt, Z. Naturforsch. 25 b, 972 [1970]. früher ${ }^{3-6}$ gefundenen Isomerenpaaren, die nur $6-22 \%$ Isomer a im Synthesegut enthalten. Wir wurden dadurch in die Lage versetzt, auch die Umwandlung des Isomeren $\mathbf{b}$ zum Isomer $\mathbf{a}$ genauer zu studieren, was bei den früher bearbeiteten Kobalt-methyl-corrinoiden infolge der ungünstigen Gleichgewichtslage nicht ohne weiteres möglich war.

Hier wird über die Darstellung der Isomeren a und $\mathbf{b}$ des Kobalt-methyl-Fakt. I b sowie über die Isomerisierungs-Versuche berichtet. Diese sollten zeigen, daß alle bisher untersuchten Isomerenpaare sich durch die gleichen strukturellen Merkmale unterscheiden und sollten gleichzeitig einen Beitrag zur Kenntnis des Isomerisierungs-Mechanismus leisten.

Da Kobalt-methyl-corrinoide in mehrere biologische Methylierungsvorgänge eingeschaltet sind ${ }^{8-10}$, wird angenommen, daß dieser Beitrag gerechtfertigt ist.

\section{Ergebnisse und Diskussion}

Wir gewannen die Isomeren $\mathbf{a}$ und $\mathbf{b}$ des Kobaltmethyl-Fakt. I b in Anlehnung an die bisherige Arbeitsweise $^{3-6}$.

7 H. Dellweg u. K. Bernhauer, Arch. Biochem. Biophysics 69, 74 [1957].

7a Nach F. Dinglinger ist Faktor Ib am C-1 der Ribose $\alpha$-glykosidisch mit $p$-Kresol verknüpft und stellt somit $\alpha$-pKresyl-cobamid vor (Privatmitt.).

8 H. A. BARKER, Biochem. J. 105, 1 [1967].

9 H. P. C. Hogenkamp, Ann. Rev. Biochem. 37, 225 [1968].

$10 \mathrm{H}$. WeISSBACH u. R. T. TAYlOR, Vitamins and Hormones 26, 395 [1968]. 
Die im Mengenverhältnis von 1:1 erhaltenen Isomeren wandern papierchromatographisch im HCN-haltigen, wasserges. sec. Butanol mit Zusatz von Essigsäure bzw. Ammoniak im Licht identisch mit nativem Fakt. I b. Die Identität mit Fakt. I b ergibt sich auch aus Elektrophorese-Versuchen im Licht bei $\mathrm{pH} \mathrm{2,5}$ und 7. Daraus ist zu schließen, daß die Methylierung, wie erwartet, nur am Kobalt erfolgte. Im Dunkeln, ohne HCN, unterscheiden sich die papierchromatographischen Beweglichkeiten der beiden Isomeren praktisch gar nicht. In Gegenwart von $\mathrm{HCN}$ und $\mathrm{NH}_{3}$ wandert das Isomer b bei Zimmertemperatur ein wenig schneller als die Dicyano-Form $\left(R_{\text {Dicyanoform }}=1,07\right)$, das Isomer a wandert gleich schnell wie die Dicyano-Form. Bei $+3{ }^{\circ} \mathrm{C}$ ist der Beweglichkeitsunterschied der beiden Isomeren noch viel geringer (praktisch nicht wahrnehmbar). Die Flecke der beiden Isomeren sind im $\mathrm{HCN}$ - und $\mathrm{NH}_{3}$ haltigen Entwickler schwarz-blau gefärbt. Die Substanzen sind unter diesen Bedingungen bei Zimmertemperatur recht stabil und es bilden sich während 24. Stdn. aus dem Isomer b nur einige Prozente Dicyano-Form (die Stabilität des Isomeren a dürfte ähnlich sein; sie läßt sich infolge der Gleichbeweglichkeit mit der Dicyano-Form auf diesem Wege nicht prüfen).

Die Absorptionsspektren der Isomeren $\mathbf{a}$ und $\mathbf{b}$ des Kobalt-methyl-Fakt. I b sind praktisch identisch mit den Spektren der entsprechenden Formen der anderen Kobalt-methyl-corrinoide ${ }^{3-6,11}$. Das Isomer $\mathbf{a}$ zeichnet sich vor allem durch die typische, gut ausgeprägte Bande bei $492 \mathrm{~nm}$ aus, die dem Isomer b fehlt (Tab. 1). Auf Grund der für die Corrinoide

\begin{tabular}{ccc}
\hline Isomer & $\begin{array}{c}\text { Wellenlänge } \\
{[\mathrm{nm}]}\end{array}$ & $\varepsilon \cdot 10^{-3}$ \\
\hline $\mathrm{a}$ & 264 & 20,4 \\
& 307 & 20,1 \\
$\mathrm{~b}$ & 492 & 12,0 \\
& 264 & 24,7 \\
& 305 & 24,9 \\
& 375 & 10,3 \\
& 464 & 10,7
\end{tabular}

Tab. 1. Absorptionsmaxima der beiden Isomeren des Kobaltmethyl-Fakt. I b in Wasser.

11 Das Spektrum des Kobalt-methyl-cobalamins entspricht diesen Bedingungen im schwach sauren $\mathrm{pH}$-Bereich, in dem die Base nicht koordiniert.

11a O. MÜller u. G. Müller, Biochem. Z. 336, 299 [1962].

$11 \mathrm{~b}$ R. A. Firth, H. A. O. Hill, J. M. Pratt, R. J. P. WilLIAMS u. W. R. JACKSON, Biochemistry 6, 2178 [1967].

12 W. FRIEDRICH, Biochem. Z. 342, 143 [1965].

13 W. Friedrich, Z. Naturforsch. 21 b, 138 [1966]. recht ungewöhnlichen Farbe der papierchromatographischen Flecke in $\mathrm{HCN}$ - und $\mathrm{NH}_{3}$-haltigem wasserges. sec. Butanol (s. o.) nahmen wir das Absorptionsspektrum der beiden Isomeren in diesem Lösungsmittel auf. Es ist für die beiden Isomeren fast identisch und zeichnet sich durch eine ausgeprägte, breite Bande bei $578 \mathrm{~nm}$ aus (Abb. 1), die für die blaue Farbe der Lösungen verantwortlich sein dürfte. Es ähnelt weitgehend dem Spektrum von Methyl-cyano-cobinamid in verd. wäßriger Cyanidlösung ${ }^{11 a, 11 b}$.

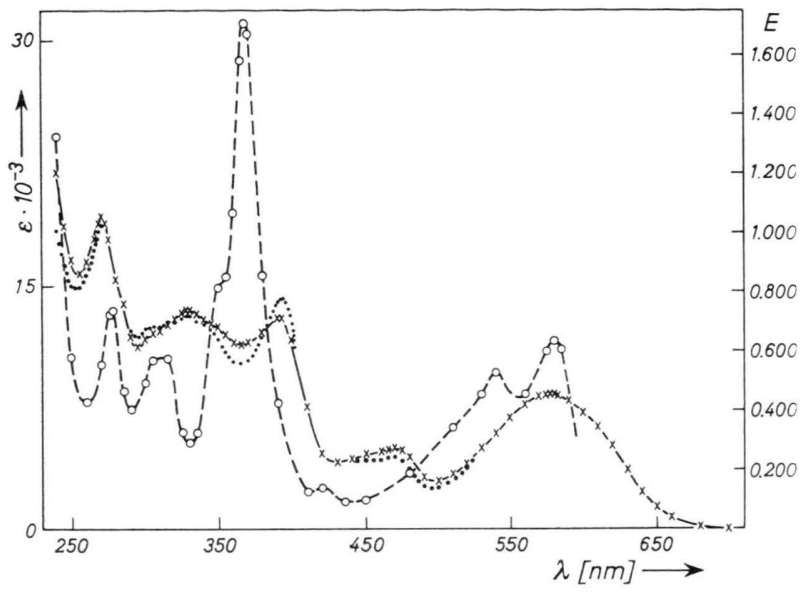

Abb. 1. Absorptionsspektrum der beiden Isomeren von Kobalt-methyl-Fakt. I b. Lösungsmittel (in ml) : sec. Butanol 50, Wasser 13, konz. $\mathrm{NH}_{3}$-Lsg. 7, 1-proz. HCN-Lsg. 5. Isomer a $-x-x-$, Isomer $\mathbf{b} \cdots$, gemeinsame Dicyano-Form -0-0-. Absorptions-Maxima für das Isomer a in $\mathrm{nm}$ (in Klammern $\left.\varepsilon \cdot 10^{-3}\right): 270(19,2) ; 330(13,5) ; 392(13) ; 470(5) ; 578(8,3)$.

Die Isomeren des Kobalt-methyl-Fakt. I b lassen sich, ähnlich wie die Isomeren der früher untersuchten Corrinoide ${ }^{3-6,12-17}$, durch Wärme bzw. durch Licht ineinander umwandeln. Wir untersuchten diese Eigenschaft quantitativ, unter Verwendung der beiden Formen, die hier in gleichen Mengen zur Verfügung standen. Wir dehnten diese Versuche auf Kobalt-methyl-cobalamin $\mathbf{a}^{3,5}$ aus. Entsprechende Versuche an Kobalt-methyl-cobyrsäure $\mathbf{a}$ wurden bereits beschrieben ${ }^{6}$. Die Ergebnisse der hier durchgeführten Isomerisierungs-Versuche lassen sich wie folgt zusammenfassen:

14 W. Friedrich, Z. Naturforsch. 21 b, 595 [1966].

15 W. Friedrich, H. OHLms, W. SandeCK u. R. Bieganowski, Z. Naturforsch. 22 b, 839 [1967].

16 W. Friedrich u. M. Moskophidis, Z. Naturforsch. 23 b, 804 [1968].

17 R. A. Firth, H. A. O. Hill, J. M. Pratt u. R. G. Thorp, J. chem. Soc. [London] Ser. A 1968, 453 


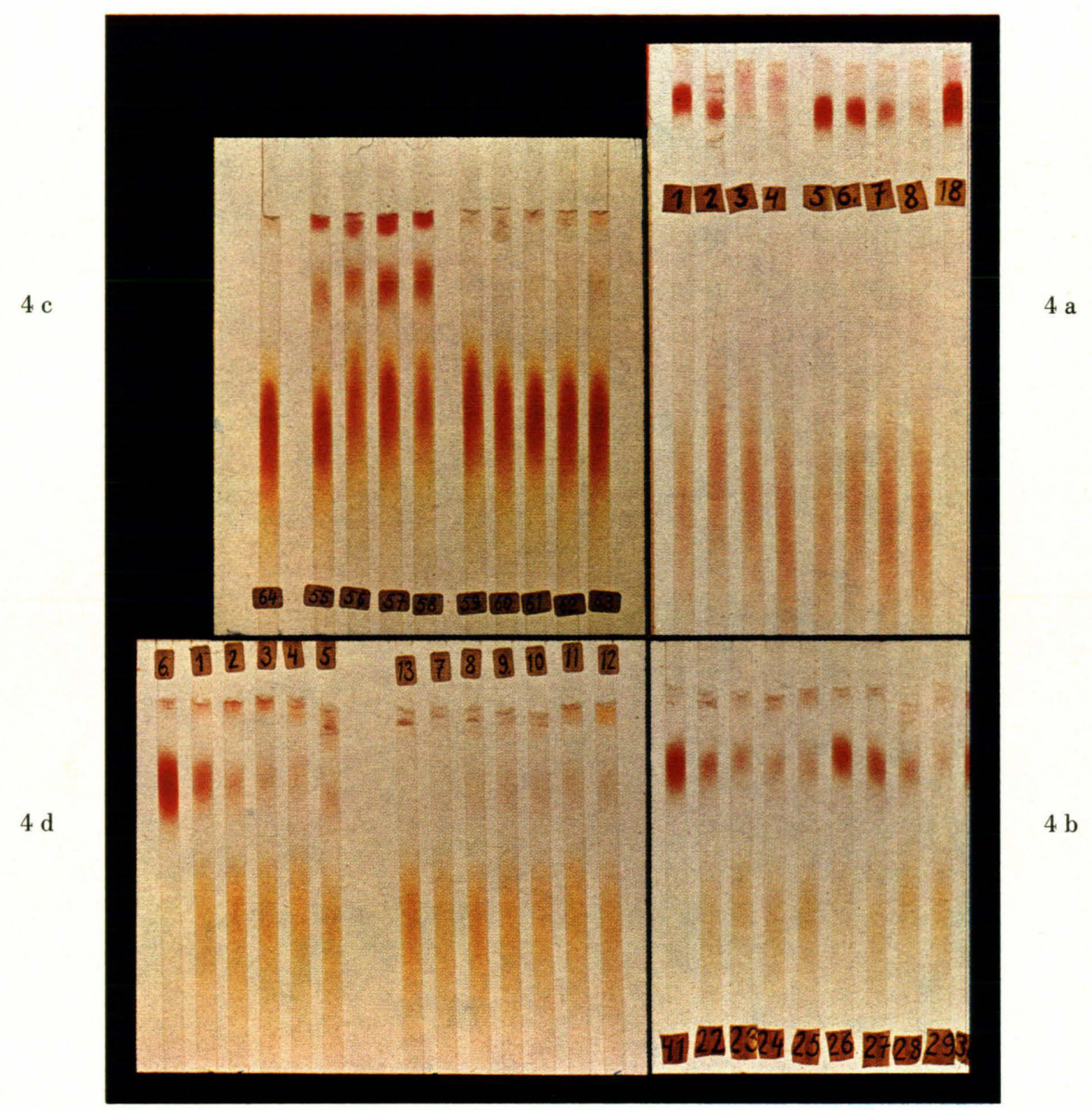

Abbn. 4 a bis 4 d. Aufnahmen von Säulenchromatogrammen einiger Isomerisierungs-Versuche (vgl. Versuchsteil) in Wasser in CO-Atmosphäre. Zeitangaben in der Reihenfolge der Proben. Säulen aus Sephadex-DEAE A-50-CN ${ }^{\ominus}$. Zonen: Dicyano-corrinoid (oben), Isomer a (Mitte), Isomer b (unten). a) Isomerisierung von Kobalt-methyl-cobalamin a zum Isomer b. Proben $1-4$ während $1 / 4 ; 1 / 2 ; 1$ und 1,5 Stdn. belichtet, Proben $5-8$ während $1 ; 2 ; 4$ und $5 \mathrm{Stdn}$. auf $80^{\circ} \mathrm{C}$ erhitzt. Probe 18 nicht behandelt. Isomer a stets im oberen Säulenteil, knapp unterhalb der violetten Zone des Dicyano-cobalamins. b) Isomerisierung von Kobalt-methyl-Fakt. I b, Isomer a zum Isomer b. Proben $22-25$ während $1 / 4 ; 1 / 2 ; 1$ und 1,5 Stdn. belichtet, Proben $26-29$ während $1 / 2 ; 1 ; 2$ und 3 Stdn. auf $80^{\circ} \mathrm{C}$ erhitzt. Probe 41 nicht behandelt. c) Isomerisierung von Kobalt-methyl-Fakt. I b, Isomer b zum Isomer a. Proben 55-58 während $1 / 4 ; 1 / 2 ; 1$ und $1,5 \mathrm{Stdn}$. belichtet, Proben $59-63$ während $1 / 2 ; 1 ; 2 ; 3$ und 4 Stdn. auf $80^{\circ} \mathrm{C}$ erhitzt. Probe 64 nicht behandelt. d) Isomerisierung von Kobalt-methyl-Fakt. I b, Isomer a (Proben 1-6) und Isomer b (Proben $7-13$ ) bei $100{ }^{\circ} \mathrm{C}$. Proben $1-5$ während ${ }^{1 / 3} ; 1 ; 1,5$; 2 und 3 Stdn., Proben $7-12$ während $1 / 3 ; 1 ; 1,5 ; 2 ; 3$ und 4 Stdn. erhitzt. Proben 6 und 13 nicht behandelt. 
1. Beim Belichten bzw. Erhitzen wäßriger Lösungen in CO-Atmosphäre findet eine rasche Isomerisierung statt. Dabei bildet sich, vor allem in der Wärme, verhältnismäßig wenig Diaquo-corrinoid ${ }^{18}$ (Abbn. $2-4^{*}$ ). Der Reaktionsablauf ist gut reproduzierbar und bei den hier untersuchten zwei Corrinoiden (Abbn. $2-4$ ) sowie bei der Kobalt-methylcobyrsäure $\mathbf{a}^{6}$ praktisch gleich.

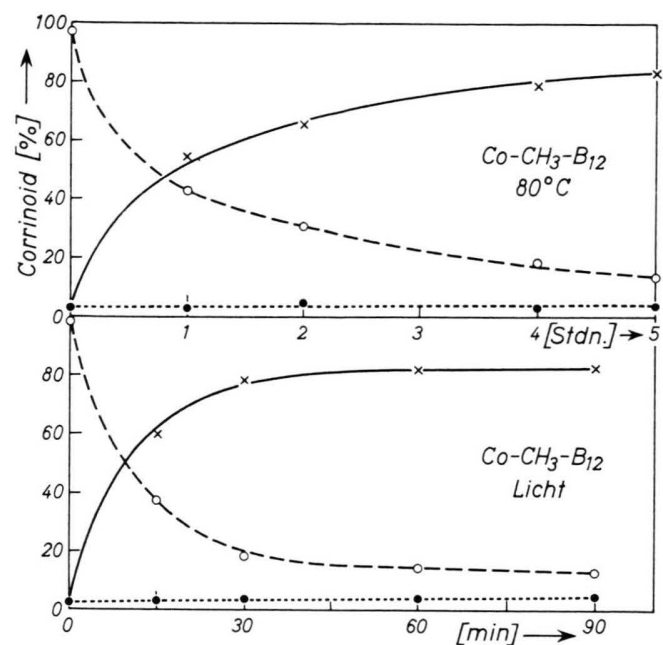

Abb. 2. Isomerisierung von Kobalt-methyl-cobalamin a (- - -) zum Kobalt-methyl-cobalamin b ( $(-)$ in Wasser in CO-Atmosphäre. Als Nebenprodukt bildet sich Aquo-cobalamin $(\cdots)$. Ampullen (vgl. Versuchsteil) auf $80^{\circ} \mathrm{C}$ erhitzt (oberes Diagramm) bzw. belichtet (unteres Diagramm). Anschließend der Inhalt jeder Ampulle mittels einer Säule $(0,65 \cdot 30 \mathrm{~cm})$ aus Sephadex-DEAE A-50-CN ${ }^{\ominus}$ chromatographiert (Entwickler: Wasser), die einzelnen Zonen nach Zerschneiden der Säulen mit 1-proz. $\mathrm{NaCl}$ eluiert und nach $\mathrm{Be}$ lichtung spektrophotometrisch gemessen. Zum gleichen Ergebnis führte die Verwendung von Säulen $(0,65 \cdot 15 \mathrm{~cm})$ aus CMCellulose (Entwickler: Wasser) ; die zu chromatographierenden Proben wurden hier zwecks Umwandlung von Aquo-cobalamin zu Cyano-cobalamin mit $0,1 \mathrm{ml}$ 1-proz. HCN versetzt.

Aus den entsprechenden Daten für Cyano-aquo-tetracarbonsäure b, welche aus Cyano-cobalamin dargestellt worden war ${ }^{12}$ (Tab.2), ist ersichtlich, daß diese bei $80{ }^{\circ} \mathrm{C}$ wesentlich schneller isomerisiert als die Kobaltmethyl-corrinoide. Dies war zu erwarten, da Tetracarbonsäure bereits bei Zimmertemperatur mit meßbarer Geschwindigkeit umgewandelt wird ${ }^{15,16}$; unter diesen Bedingungen sind Kobalt-methyl-corrinoid-Lösungen im Dunkeln stabil.

2. In $\mathrm{O}_{2}$-Atmosphäre findet beim Belichten wäßriger Lösungen keine Isomerisierung statt, dafür bildet sich viel Aquo- bzw. Diaquo-corrinoid. Beim Erhitzen in $\mathrm{O}_{2}$-Atmosphäre ist die Isomerisierung

18 Dieses wird während der Chromatographie in der Sephadex-DEAE A-50-CN ${ }^{\ominus}$-Säule zur Dicyano-Form umgewandelt und im obersten Säulenteil festgehalten.

* Abbn. 4 a-4 d s. Tafel S. 980 a.

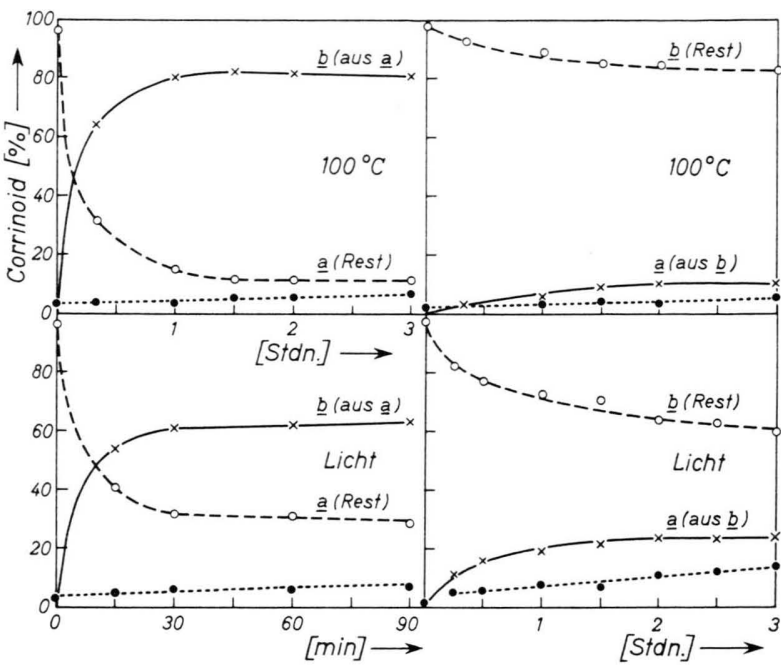

Abb. 3. Isomerisierung der Formen a und $\mathbf{b}$ des Kobalt-methyl-Frakt. I b in Wasser in CO-Atmosphäre. Erhitzte bzw. belichtete Proben (vgl. Versuchsteil) mittels Sephadex-DEAE A-50-CN ${ }^{\ominus}$ (Säulen 0,65.20 cm) chromatographiert (Entwickler: Wasser). Nach Zerschneiden der Säulen Elution der Zonen mit 1-proz. $\mathrm{NaCl}$ (enthaltend etwas $\mathrm{KCN}$ ) und spektrophotometrische Auswertung bei $367 \mathrm{~nm}$. Eingesetztes Corrinoid (- - ) ergab stets neben dem gebildeten Isomer $(-)$ eine gewisse Menge an Diaquo-Form $(\cdots)$. Ausgehend vom Isomer a (linke Diagramme) bzw. vom Isomer b (rechte Diagramme) wird das Gleichgewicht bei $100^{\circ} \mathrm{C}$ (obere Diagramme) innerhalb 1,5 bis 2 Stdn., beim Belichten $\left(20^{\circ} \mathrm{C}\right.$, $15 \mathrm{~W}, 39 \mathrm{~cm}$; untere Diagramme) innerhalb 1 bis $2 \mathrm{Stdn}$. erreicht.

eindeutig, jedoch im allgemeinen viel langsamer als unter CO (Tab. 2). In Stickstoff-Atmosphäre im Licht verläuft die Isomerisierung langsam; sie ist etwas rascher, wenn das Gas mittels alkalischer Pyrogallol-Lösung nachgereinigt wurde (Tab. 2).

3. Erhitzt oder belichtet man in CO-Atmosphäre eine wäßrige Lösung von Kobalt-methyl-Fakt. I b in Gegenwart von Aquo-cobalamin, dann gewinnt man Kobalt-methyl-cobalamin; in $\mathrm{O}_{2}$-Atmosphäre ist die Ausbeute an Kobalt-methyl-cobalamin sehr niedrig (Tab. 2).

4. Isomerisierung von Kobalt-methyl-Fakt. I b, Isomer $\mathbf{a}$, durch Licht oder Wärme unter $\mathrm{CO}$ in Wasser, in $0,05 \mathrm{~N}$ Essigsäure bzw. in $0,05 \mathrm{M}$ $\mathrm{NH}_{4} \mathrm{HCO}_{3}$ verläuft mit praktisch gleicher Geschwindigkeit, diese dürfte also $\mathrm{pH}$-unabhängig sein.

5. Wie Versuche an den beiden Isomeren des Kobalt-methyl-Fakt. I b zeigten, werden in alkoholischen Medien (Methanol, Äthanol, Isopropanol) gelöste Corrinoide weder in $\mathrm{CO}$ - noch in $\mathrm{O}_{2}$-Atmosphäre, weder durch Licht noch durch Wärme isomerisiert. Belichtung in Methanol ergab meist mehr Diaquo-corrinoid als Belichtung in Wasser. 


\begin{tabular}{|c|c|c|c|c|c|c|}
\hline \multirow{2}{*}{ Corrinoid } & \multirow{2}{*}{$\begin{array}{l}\text { Lösungs- } \\
\text { mittel }\end{array}$} & \multirow[t]{2}{*}{ Gas } & \multicolumn{2}{|c|}{ Behandlung } & \multicolumn{2}{|c|}{ Gebildetes Isomer } \\
\hline & & & $\begin{array}{l}\text { Warme, } \\
\text { bzw. Licht }\end{array}$ & $\begin{array}{l}\text { Zelt, } \\
{[\mathrm{h}]}\end{array}$ & Art & {$[\%]^{*}$} \\
\hline $\mathrm{Co}-\mathrm{CH}_{3}-\mathrm{B}_{12}$ a & Wasser & $\begin{array}{c}\mathrm{O}_{2} \\
\mathrm{~N}_{2} \\
\mathrm{~N}_{2} \text { ger.*** }\end{array}$ & $\begin{array}{l}90^{\circ} \mathrm{C} \\
\text { Licht } * *\end{array}$ & $\begin{array}{l}4 \\
1 / 2 \\
1 \\
1 / 2 \\
1\end{array}$ & $\mathrm{Co}-\mathrm{CH}_{3}-\mathrm{B}_{12} \mathbf{b}$ & $\begin{array}{r}8 \\
5 \\
26 \\
7 \\
37\end{array}$ \\
\hline $\mathrm{Co}-\mathrm{CH}_{3}$-Fakt. Ib, Isomer a & & $\mathrm{O}_{2}$ & & $1^{1 / 2}$ & $\begin{array}{l}\mathrm{Co}-\mathrm{CH}_{3} \text {-Fakt. Ib, } \\
\text { Isomer b }\end{array}$ & 0,0 \\
\hline $\begin{array}{l}\text { Co- } \mathrm{CH}_{3} \text {-Fakt. Ib, Is. a und Aquo- } \\
\text { cobalamin }\end{array}$ & & $\mathrm{CO}$ & $\begin{array}{l}80^{\circ} \mathrm{C} \\
\text { Licht** } \\
80^{\circ} \mathrm{C}\end{array}$ & $\begin{array}{l}3 \\
3 \\
4\end{array}$ & $\mathrm{Co}-\mathrm{CH}_{3}-\mathrm{B}_{12} \mathbf{b}$ & $\begin{array}{r}7 \\
14 \\
40\end{array}$ \\
\hline Cyano-aquo-tetracarbonsäure b (aus $\mathrm{B}_{12}$ ) & $\begin{array}{l}0,05-\mathrm{v} \\
\text { Essigsäure }\end{array}$ & $\begin{array}{l}\mathrm{O}_{2} \\
\mathrm{CO}\end{array}$ & & $\begin{array}{l}4 \\
1 / 12 \\
1 / 6 \\
1 / 3 \\
2 / 3\end{array}$ & $\begin{array}{l}\mathrm{CN}-\mathrm{H}_{2} \mathrm{O} \text {-Tetra- } \\
\text { carbonsäure a }\end{array}$ & $\begin{array}{l}\text { Spur } \\
32,3 \\
40,8 \\
43,8 \\
44,3\end{array}$ \\
\hline
\end{tabular}

Tab. 2. Isomerisierungs- bzw. Methylübertragungs-Reaktionen an Corrinoiden unter verschiedenen Bedingungen. Versuche in $1 \mathrm{ml}$ fassenden Glasampullen. Je Ampulle 0,4 ml Lösung, enthaltend ca. $400 \mathrm{nMol}$ Corrinoid oder je ca. $400 \mathrm{nMol}$ zweier Corrinoide. Ampulleninhalt auf die übliche Weise behandelt und chromatographisch analysiert (s. Versuchsteil). * Bezogen auf die Summe der Corrinoide. ${ }^{* *} 15 \mathrm{~W}, 39 \mathrm{~cm}, 20^{\circ} \mathrm{C}$. *** Gereinigt mittels alkalischer Pyrogallollösung.

6. Obwohl bei der Synthese die beiden Isomeren des Kobalt-methyl-Fakt. I b im Mengenverhältnis von $1: 1$ gebildet werden, werden beim Belichten bei $20{ }^{\circ} \mathrm{C}$ stets ca. $30 \%$ Isomer a neben ca. $70 \%$ Isomer b erhalten, unabhängig davon, von welchem Isomeren ausgegangen wurde. Beim Erhitzen auf $100{ }^{\circ} \mathrm{C}^{19}$ erhält man, ausgehend analog von den beiden Formen, nur ca. 11\% Isomer a neben ca. 89\% Isomer b, eine Zusammensetzung, die etwa der der übrigen in dieser Richtung untersuchten Kobaltmethyl-corrinoide entspricht (Abbn. 3 und 4d).

Die meisten der hier beschriebenen Eigenschaften sowie der Charakter der Absorptionsspektren sind allen bisher untersuchten Isomerenpaaren der Kobalt-methyl-corrinoide gemeinsam und weichen von denen der Isomerenpaare der Cyano-aquo-corrinoide ${ }^{15,16}$ anscheinend nur quantitativ $\mathrm{ab}^{6}$.

Es ist noch nicht klar, worauf das Verhalten des Kobalt-methyl-Fakt. I b hinsichtlich der Isomerenverteilung zurückzuführen ist. Die drei verschiedenen Mengenverhältnisse, die bei Synthese, Belichtung und Erwärmung beobachtet wurden, sind vielleicht auf die Unterschiede in Milieu und Temperatur (Synthese: in 10-proz. $\mathrm{NH}_{4} \mathrm{Cl}$; Belichtung: in Wasser, $20^{\circ} \mathrm{C}$; Erwärmung: in Wasser, $100^{\circ} \mathrm{C}$ )

19 Bei $80^{\circ} \mathrm{C}$ während $4 \mathrm{Stdn}$. konnte das Gleichgewicht nicht erreicht werden. Vgl. Abbn. $4 \mathrm{~b}$ und $4 \mathrm{c}$.

$20 \mathrm{Zu}$ diesem Problem wird demnächst ausführlich Stellung genommen werden (W. FRIEDRICH, in Vorbereitung).

21 R. Yamada, S. Shimizu u. S. Fukui, Biochim. biophysica Acta [Amsterdam] 124, 195, 197 [1966]. zurückzuführen. Die Tatsache, daß in alkoholischem Milieu keine Isomerisierung abläuft, spricht dafür, daß diese auf Wassermoleküle angewiesen ist ${ }^{20}$.

Mit der Stimulierung anaerober Photolyse des Kobalt-methyl-cobalamins durch Alkohole befaßten sich YAMADA u. a. ${ }^{21}$. Die Autoren vertreten die Ansicht, daß die als Radikal abgespaltene Methylgruppe durch den Entzug eines H-Atoms vom Alkoholmolekül stabilisiert wird. Die relativ hohe Stabilität von Kobalt-methyl-corrinoiden gegenüber Photolyse in $\mathrm{O}_{2}$-freien wäßrigen Medien ${ }^{21,22}$ ist auf mangelhafte Stabilisierungsmöglichkeit des gebildeten Methylradikals zurückzuführen. Dieses ist dadurch in der Lage, durch Rekombination das ursprüngliche Kobalt-methyl-corrinoid zu bilden ${ }^{21,23}$. Diese Rekombination wird eventuell durch den „Käfigeffekt" des Wassermantels erleichtert, der das photolysierte Molekül (oder eine Molekülgruppe?) umgibt ${ }^{23}$. Diese Vorstellung könnte nach unserer Auffassung die Basis für den Isomerisierungs- und Methylübertragungs-Vorgang sein (unabhängig davon, ob die Co-C-Bindung homolytisch oder heterolytisch gespalten wird), da bei der Rekombination auch die andere Molekülseite bzw. ein anderes Corrinoid-Molekül reagieren dürfte.

22 D. Dolphin, A. W. Johnson, R. Rodrigo u. N. Shaw, Pure appl. Chem. 7, 539 [1963].

23 H. P. C. Hogenkamp, Biochemistry 5, 417 [1966].

23a Lampe Philips A5, matt. 
Die hier zur Isomerisierung von Kobalt-methylcorrinoiden benötigten Lichtmengen $(15 \mathrm{~W}, 39 \mathrm{~cm})^{23 \mathrm{a}}$ sind viel geringer als die von anderen Autoren zur Abspaltung der Methylgruppe (unter Bildung von Aquo-corrinoid) in wäßrigen Medien verwendeten (z. B. $375 \mathrm{~W}, 20 \mathrm{~cm}^{23}$ ). Daraus ist ersichtlich, daß bereits sehr geringe Energiemengen zur weitgehenden Lockerung der Kobalt-methyl-Bindung genügen, vor allem in Gegenwart von Wasser.

Die Tatsache der Nicht-Isomerisierung in Alkoholen läßt sich durch die geschilderte Reaktion der Methylradikale mit Alkoholmolekülen nicht erklären, da diese Reaktion viel langsamer abläuft als die Isomerisierung in Wasser.

Die Rolle des Kohlenmonoxides bei den Isomerisierungs-Reaktionen wurde bereits besprochen ${ }^{6}$. CO verändert die Corrinoide unter den hier eingehaltenen Bedingungen nicht und dürfte nur als Katalysator wirken, da die Isomerisierung auch unter $\mathrm{O}_{2}$ abläuft, vor allem beim Erhitzen ${ }^{6}$ (Tab. 2).

FIRTH u. a. ${ }^{17}$ nehmen folgenden IsomerisierungsMechanismus der Cyano-aquo-corrinoide an: die - in Spuren stets vorhandene - schweflige Säure in Wasser gelöster Proben koordiniert mit Kobalt unter Freisetzung von Blausäure. Diese bildet mit unversehrten Cyano-aquo-Molekülen die Dicyano-Form, welche dann in HCN und Cyano-aquo-corrinoid zerfällt, wobei mit spezifischer Wahrscheinlichkeit das Isomer der Ausgangsform entsteht. Eine Isomerisierung von Kobaltmethyl-corrinoiden - die sich übrigens nach diesem Schema nicht erklären ließe - wurde von den oben genannten Autoren nicht beobachtet (es wurde allerdings nur dünnschicht-chromatographischer Nachweis benutzt) ${ }^{17}, 24$

Nach dem zitierten Mechanismus ${ }^{17}$ müßte die Isomerisierungs-Geschwindigkeit weitgehend von der $\mathrm{SO}_{2}$ Konzentration der Corrinoidlösung abhängen. Da diese Konzentration eine zufällige und somit stark variierende Größe ist, müßte auch die Isomerisierungs-Geschwindigkeit entsprechend variieren. Das Experiment zeigt jedoch eine gute Reproduzierbarkeit des Isomerisierungsvorgangs 15,16

Der vorgeschlagene Mechanismus ${ }^{17}$ kann folgende Befunde ${ }^{25}$ nicht erklären : beim Erwärmen einer wäßrigen Lösung, enthaltend Cyano-aquo-tetracarbonsäure ${ }^{12,15}$ und Aquo-cobalamin, werden in präparativem Maßstab Cyano-cobalamin und Diaquo-tetracarbonsäure, jedoch praktisch keine Sulfito-tetracarbonsäure gebildet; nach dem vorgeschlagenen Mechanismus ${ }^{17}$ müßte sich jedoch für jedes Molekül Cyano-cobalamin ein Molekül Sulfito-tetracarbonsäure gebildet haben. Auch wenn dieser Mechanismus ${ }^{17}$ auf die Cyono-aquocorrinoide teilweise anwendbar wäre, was ja durch-

24 R. A. Firth, H. A. O. Hill, J. M. Pratt u. R. G. Thorp, Analyt. Biochem. [New York] 23, 429 [1968]. aus möglich ist, ist seine Gültigkeit im Bereich der Kobalt-methyl-corrinoide ausgeschlossen. Andererseits spricht die wesentliche Stimulierung der Isomerisierung ${ }^{15}, 16$ und der CN-Übertragung ${ }^{25}$ durch $\mathrm{CO}$ für einen wenigstens teilweise gemeinsamen Mechanismus ${ }^{6}$ im Bereich der Cyano-aquo- und der Kobalt-methylcorrinoide.

\section{Experimenteller Teil}

\section{Arbeitsbedingungen}

Die Versuche wurden bei weitgehendst reduziertem weißem Licht durchgeführt (eine Ausnahme bilden die Belichtungsversuche).

\section{Partialsynthese der Isomeren a und $\mathbf{b}$ des Kobalt- methyl-Fakt. Ib}

In einem $50 \mathrm{ml}$ fassenden Meßkolben wurden $2 \mathrm{~g}$ $\mathrm{NH}_{4} \mathrm{Cl}$, $1 \mathrm{~g}$ Zinkpulver und $100 \mathrm{mg}$ Faktor Ib (Reinheitsgrad ca. 60\%) in $20 \mathrm{ml}$ Wasser unter Eiskühlung während $30 \mathrm{~min}$ mit Wasserstoff begast und mit $1 \mathrm{ml}$ Methyljodid, gelöst in $4 \mathrm{ml} \mathrm{Methanol,} \mathrm{versetzt.} \mathrm{Nach}$ weiteren 10 min wurde die Begasung beendet und das Gefäß während 10 min verschlossen gehalten. Es folgten Phenolextraktion (mit einem Phenol-o-Dichlorbenzol-Gemisch) und Chromatographie mit Hilfe einer Säule $(2,9 \cdot 20 \mathrm{~cm})$ aus Sephadex-DEAE A-50-CN ${ }^{\ominus}$ bei $0{ }^{\circ} \mathrm{C}^{6}$. Beim Entwickeln mit Wasser bewegten sich nur zwei sehr starke, stahlgraue, fast schwarze, sehr gut getrennte Zonen der Isomeren b (schnell) und a (langsam). Nach der Chromatographie verblieben in der Säule zwei sehr schwache violette Zonen, eine schwache orangegelbe Zone (nicht licht-empfindlich) und - ganz oben - etwas Dicyano-Fakt. Ib. Ausbeute (spektrophotometrisch gemessen) : $22 \mathrm{mg}$ Isomer a und $21,5 \mathrm{mg}$ Isomer b.

\section{Isomerisierungs-Versuche}

Man füllte eine Lösung, enthaltend ca. $400 \mathrm{nMol}$ eines Corrinoides bzw. ein Gemisch, enthaltend je ca. $400 \mathrm{nMol}$ zweier Corrinoide in 0,4 ml Lösungsmittel, in $1 \mathrm{ml}$ fassende farblose Glasampullen. Der Ampulleninhalt wurde mit $\mathrm{CO}, \mathrm{N}_{2}$ bzw. $\mathrm{O}_{2}$ gesättigt ${ }^{26}$, die Ampullen mit einer Sparflamme zugeschmolzen und erhitzt (auf $80{ }^{\circ} \mathrm{C}, 90^{\circ} \mathrm{C}$ bzw. $100^{\circ} \mathrm{C}$ ) oder belichtet $(15 \mathrm{~W}$, $39 \mathrm{~cm}, 20^{\circ} \mathrm{C}$ ). Die Corrinoide wurden anschließend mit Hilfe von Säulen aus Sephadex-DEAE A-50-CN ${ }^{\ominus}$ $(0,65 \cdot 20-30 \mathrm{~cm})^{6} \mathrm{bzw}$. aus CM-Cellulose $(0,65 \cdot 15$ $\mathrm{cm})$ chromatographiert, die gebildeten Zonen mit 1-proz. $\mathrm{NaCl}$ eluiert und spektrophotometrisch ausgewertet. Zur Identifizierung der einzelnen Zonen dienten das Absorptionsspektrum und die Papierchromatographie.

Wir danken der Deutschen Forschungsgemeinschaft und der Hoffmann-LaRoche AG, Basel, für die Unterstützung dieser Arbeit.

\footnotetext{
25 W. Friedrich u. R. Bieganowski, Z. Naturforsch. 22 b, 741 [1967].

${ }^{26}$ Zum Reinheitsgrad der verwendeten Gase vgl. 1. c. ${ }^{25}$.
} 